\title{
Assessing the Lockdown Effects on Air Quality During COVID-19 Era
}

\author{
Ioannis Kavouras ${ }^{\text {a }}$ Eftychios Protopapadakis a Maria Kaselimi ${ }^{\text {a }}$ Emmanuel Sardis ${ }^{\text {a }}$ and \\ Nikolaos Doulamis ${ }^{\text {a }}$ \\ ${ }^{a}$ National Technical University of Athens, Zografou Campus 9, Iroon Polytechniou str, \\ Athens, 15780, Greece, ikavouras@mail.ntua.gr, eftprot@mail.ntua.gr, \\ mkaselimi@mail.ntua.gr,ndoulam@cs.ntua.gr
}

\begin{abstract}
.
In this work we investigate the short-term variations in air quality emissions, attributed to the prevention measures, applied in different cities, to mitigate the COVID-19 spread. In particular, we emphasize on the concentration effects regarding specific pollutant gases, such as carbon monoxide $(\mathrm{CO})$, ozone $\left(\mathrm{O}_{3}\right)$, nitrogen dioxide $\left(\mathrm{NO}_{2}\right)$ and sulphur dioxide $\left(\mathrm{SO}_{2}\right)$. The assessment of the impact of lockdown on air quality focused on four European Cities (Athens, Gladsaxe, Lodz and Rome). Available data on pollutant factors were obtained using global satellite observations. The level of the employed prevention measures is employed using the Oxford COVID-19 Government Response Tracker. The second part of the analysis employed a variety of machine learning tools, utilized for estimating the concentration of each pollutant, two days ahead. The results showed that a weak to moderate correlation exists between the corresponding measures and the pollutant factors and that it is possible to create models which can predict the behaviour of the pollutant gases under daily human activities.
\end{abstract}

Keywords. environmental quality, air pollution, air quality levels, neural networks, COVID-19, machine learning, dynamic time warping

\section{Introduction}

By the end of January 2020, authorities, worldwide, introduced a series of measures, in order to reduce the transmissibility of the COVID-19 virus epidemic [2]. These measures include international travel controls (e.g. travel prohibition between countries), restrictions in internal movements (e.g. restrictions in transportation between municipalities), public event cancellations (e.g. closure of live concerts), restriction gatherings (e.g. limiting the amount of people over an area), closure of public transportation, closure of schools, stay home requirements (e.g. move outside the house only for work, emergency, etc) and closure of workplaces (e.g. closure of industries / commercial sector) [3].

The response measures appeared to have an impact on the Air Quality Levels (AQL) in cities. Indicative examples involve countries such as China [4], India [5], Brazil [6] and Spain [7]. However, there are no clear indication regarding the correlation between each major pollutant factor, i.e. Carbon Monoxide $(\mathrm{CO})$, Ozone $\left(\mathrm{O}_{3}\right)$, Nitrogen Dioxide $\left(\mathrm{NO}_{2}\right)$ and Sulphur Dioxide $\left(\mathrm{SO}_{2}\right)$, and the applied measures. The general trends indi- 
cate that applied measures improved the AQL. There are various environmental models, based on simulations [8,9], indicating the causality between specific actions and AQL. Nevertheless, the models have not been sufficiently tested in real life. Thus, the lockdown, worldwide, created a unique opportunity for researching the significance of traffic reduction in AQL changes.

In this work we will investigate the correlation between the applied lockdown measures and the four major pollutant factors $\left(\mathrm{CO}, \mathrm{O}_{3}, \mathrm{NO}_{2}\right.$ and $\left.\mathrm{SO}_{2}\right)$ for a total of four European Cities - Athens (Greece), Gladsaxe (Denmark), Lodz (Poland) and Rome (Italy) - during COVID-19 period. In particular, we focus on the identification of each measure and what scale affects each of the pollutant factors. This will be achieved by a variety of statistical correlation methods, where we will test the relation between each measure, at a time, and the density of each pollutant factor, for a yearly period divided by two days average values. The pollutant factor densities will be calculated using the Sentinel-5 TROPOMI satellite images of a two day period, clipped in a squared area of radius $0.25^{\circ}$ around the center of each city.

Also, a variety of machine learning methods will be trained using the most correlated COVID-19 measures and the densities of each one of the most dangerous pollutant gases $\left(\mathrm{CO}, \mathrm{O}_{3}, \mathrm{NO}_{2}, \mathrm{SO}_{2}\right)$. A different model will be created, for each of the pollutant factors. In total, four models per machine learning methods will be trained. As input we will use a two days period COVID-19 measures and each pollutant's mean density. The output will be each pollutant's mean density, two days ahead.

\section{Related Work}

The multiple and synchronized applied measures (lockdown) for the minimization of COVID-19 transmissibility, provided the opportunity for an in depth research of the impact of industrial activities closure and traffic volume reduction, in cities, and AQL. During and after the lockdown, in China, researches proposed a reductive trend in $C O$, $\mathrm{O}_{3}, \mathrm{NO}_{2}, \mathrm{SO}_{2}, \mathrm{PM}_{10}$ and $\mathrm{PM}_{2.5}$ densities [10,11,12]. Indicative example, consists the Hubei region, in which during the lockdown period in January and February, 2020, the Angstrom Exponent (AE) and the Aerosol Optical Depth (AOD) increased and decreased respectively by $29.4 \%$ and $39.2 \%$ (only in Wuhan they increased and decreased by $45.3 \%$ and $31.0 \%$ ) [13].

Another, significance example, includes India, in which has been observed reduction, during and after the lockdown, in critical pollutant factors [14,15,16]. A comparison in air pollution changes between India and China, before and after the lockdown, described in the work of [17]. Real-time measured densities of $P M_{2.5}$ and $\mathrm{NO}_{2}$ are used for a total of 12 megacities in India and China (6 megacities from each country). An Air Quality Index (AQI) is calculated, for each density, before, during and after the lockdown, which resulted to a reduction of $45.25 \%\left(A Q I_{P_{2.5}}\right)$ and $64.65 \%\left(A Q I_{N_{2}}\right)$ in China and $37.42 \%\left(A Q I_{P M_{2.5}}\right)$ and $65.80 \%\left(A Q I_{N_{2}}\right)$ in India after the lockdown.

Other related works, include, the [18], in which the densities of $\mathrm{CO}, \mathrm{O}_{3}, \mathrm{NO}_{2}$ and $\mathrm{SO}_{2}$ were predicted one week ahead, while, also, an AQI was calculated, which estimated that in countries, such as India, where the lockdown measures were stricter, the air quality improved, while on the other hand in countries like Australia where the lockdown measures, weren't so strict the air quality followed an incremented trend. In Madrid and 
Barcelona (Spain) [19] the concentration of $\mathrm{NO}_{2}$ reduced $62 \%$ and $50 \%$, respectively, after a 75\% in traffic volume. In Baghdad (Iraq) [20] the daily densities of $\mathrm{NO}_{2}, \mathrm{O}_{3}$, $P M_{2.5}$ and $P M_{10}$ used before and during the lockdown, in addition to the AQI for the same time period.

\section{Proposed methodology}

In this work we focus on two research topics: a) how each of the specific prevention measures is related on each of the pollutant factor levels, and b) to what extent we could estimate (forecast) the trend of each pollutant level, when using as input the current level, plus the prevention measures. On the one hand, the estimation of the correlation between COVID-19 measures and pollutant factors can be evaluated using various case-specific techniques [22]. On the other hand, the estimation of pollutant factors' levels few days ahead, entails to traditional regression problem [21].

Let us denote as $\mathbf{x}=\left[x_{1}, \ldots, x_{n}\right]$, a set of predictive factors, involving prevention measures magnitude and current pollution levels, and $\mathbf{y}$ as the investigated pollutant factor. We will try to estimate a predictive function $f(\mathbf{x}) \longrightarrow \hat{\mathbf{y}}$, where $\hat{y}$ stands for the specific pollutant factor's level, two days ahead. Practically, we would like to have values as close as possible to actual ones, i.e. $\hat{\mathbf{y}} \simeq \mathbf{y}[23]$.

The input data, is a combination of: a) the densities of the most harmful pollutant gases $\left(\mathrm{CO}, \mathrm{O}_{3}, \mathrm{NO}_{2}\right.$ and $\mathrm{SO}_{2}$ ), day-by-day period, and b) the magnitude of the applied prevention measure ranging from 0 (no measure applied) to 1 (the strictest form of the measure). Experiments rung in a total of 4 European Cities (Athens, Gladsaxe, Lodz, Rome). The adopted COVID-19 mitigation strategies, considered as predictive factors, are explained in the following lines.

Restrictions of Internal Movement (RE_IN_MOV) refers to the transportation between municipalities. International Travel Controls (IN_TR_CON) include any measure which restricts/prohibits the transportation between countries. Cancellation of Public Events (CA_PUB_EV) involves the cancellation of all public events, locally (e.g. football matches, concerts) or worldwide (e.g. Olympic Games in Japan, Euro-vision). Restriction in Gatherings (RE_GAT) is a general description for all measures that restricts more than a number of people be together, in the same place. Close Public Transportation (C_PUB_TRAN) includes the measures taken about the closure of public transportation (e.g. busses, metro). School Closures (C_SCHOOL) describe any measure involving the education system (e.g. school closure, distance learning, etc). Stay at Home Requirement(STAY_HOME_R) is considered the most intense measure, since it penalized any movement outside home, without a specified reason (e.g. visit to the doctor, pharmacy, provide assistance to relative, etc). Workplace Closures (C_WORKPLACE) refer to both the industrial activity and the commercial sector.

The COVID-19 dataset has been downloaded from Our World in Data (https: //ourworldindata.org/policy-responses-covid) [3], which been updated daily. The densities of the four pollutant factors have been downloaded from the Sentinel5/TROPOMI dataset of Google Earth Engine [24], in a day-by-day period, with 50m spatial analysis. 


\subsection{Investigating patterns similarity}

As a first step, two statistical methods have been used. The core idea was to identify whether there is high correlation between the investigated pollutant factor and any of the applied prevention measures. These statistical measures are known as a) Pearson correlation and b) Dynamic Time Wrapping (DTW).

Pearson correlation estimates how related two signals are, using the best fit line approach. This method calculates three values, which refers to correlation (r), coefficient of determination $\left(R^{2}\right)$ and hypothesis (p). The $\mathrm{r}$ can be any value between -1 and 1 (the sign shows the direction) and absolute value can be translated as:

- $0.00-0.09=$ No Correlation

- $0.10-0.39=$ Weak Correlation

- $0.40-0.69=$ Moderate Correlation

- $0.70-0.89$ = Strong Correlation

- $0.90-1.00$ = Very Strong Correlation

The $R^{2}$ can be any value between $[0,1]$ and can be multiplied by 100.0 to express the percent of affection between correlated objects. The $\mathrm{p}$ can be any value between range $[0$, 1] representing the probability that this data would have arisen if the null hypothesis were true (Null hypothesis: There is no correlation between A and B in the overall population $r=0$, Alternative hypothesis: There is a correlation between A and B in the overall population $r \neq 0)$. [25,26]

DTW is a technique used for calculating the alignment between two given (timedependent) signals, under certain restrictions. The sequences are warped in a non-linear fashion to match each other. DTW, originally, has been used for the comparison of different speech patterns in automatic speech recognition. [27]

\subsection{Machine Learning Tools for Extracting Environmental Impact}

Various machine approaches have been evaluated. These approaches range from traditional shallow learning techniques like k-nearest neighbors to more complex approaches as deep neural networks. The outcomes indicate that, for the problem at hand, and due to the low feature space dimensional, traditional approaches are sufficient for robust pollutant factors level estimations.

Deep Neural Network (DNN): A DNN method is a feed forward network that consists of multiple hidden layers. For the purpose of this research we used for input a set of predictive values $\mathbf{x}=\left[x_{1}, \ldots, x_{n}\right]$, and as output $\mathbf{y}=\left[y_{1}, y_{2}\right]$. The architecture, also, consists of three hidden layers $\left(L_{1}, L_{2}, L_{3}\right)$, with sizes 20, 10 and 20 accordingly. As activation function between the hidden layers, the SELU (Scaled Exponential Linear Units) has been chosen, and for the output layer the sigmoid.

Long Short-Term Memory (LSTM) networks are a type of recurrent neural network (RNN) capable of learning order dependence in sequence prediction problems. The two technical problems, of RNN, overcome by LSTMs are vanishing gradients and exploding gradients, both related to how the network is trained. Hyperparameter optimization is feasible through Bayesian optimization schemes. [36]

Decision Tree Regressor (DTR): DTR method's core idea lies in sub-dividing the space into smaller regions and then fit simple models to them. Practically, every internal 
node tests an attribute and according to which cell the path, on the branches, ends, an average value over the available observations is calculated [29]. The main parameter a DTR need for running appropriately is the maximum depth of the tree. In our case we used a maximumDepth $=5$.

Random Forest Regression(RFR) RFR is an alternative of the DTR (in case of regression can be called regression tree - RTR - as well) methodology. In this case the concept of the DTR(RTR) can be extended using the power of contemporary computers to generate hundreds of DTRs(RTRs), simultaneously, known as random forests. The final prediction can be estimated by the smallest MSE calculated from the average of the various DTRs(RTRs)[33,34]. RFR takes as parameters the maximum depth and a random state. We used maximumDepth $=5$ and randomState $=2$.

K-Nearest Neighbor (K-NN): K-NN is a non parametric supervised machine learning method, which makes no assumptions for the underlying data distribution. In this method, for every instance, the distances (usually Euclidean) between a $x_{i}$ feature and all features of the training set, are calculated. Then the k-nearest neighbors are selected and the $x_{i}$ feature is classified with the most frequent class among the k-nearest neighbors [30].

Linear Regression (LReg) can be expressed as the statistical method applied to a dataset to quantify and define the relation between the considered features. LReg can be used for forecasting. We have, also, consider other types of linear models, fitted by minimizing a regularized empirical loss with Stochastic Gradient Descent (Multi O/P GBR - MGBR), or by fitting a regressor on the original dataset and then fits additional copies of the regressor on the same dataset but where the weights of instances are adjusted according to the error of the current prediction. As such, subsequent regressors focus more on difficult cases (Multi O/P AdaB - MAdaB) [32]. In both training methods (MGBR and MAdab) we used estimators $=5$.

Lasso is a modification of linear regression, where the model is penalized for the sum of absolute values of the weights. Thus, the absolute values of weight will be (in general) reduced, and many will tend to be zeros. Lasso algorithm can be explained further by the LARS algorithm [31]. Ridge regression is another method for modeling the connection between a depended scalar variable with one or more explanatory variables. It can be seen as a step further to Lasso. Ridge regression penalizes the model for the sum of squared value of the weights. Thus, the weights not only tend to have smaller absolute values, but also really tend to penalize the extremes of the weights, resulting in a group of weights that are more evenly distributed [35].

\section{Experimental Setup}

\subsection{Dataset description}

The dataset is composed by 4 European Cities (Athens, Gladsaxe, Lodz, Rome). For each city has been calculated the measures per day-by-day period from the 1st of January, 2020, as the first day and 31st of December, 2020, as the last. This methodology splits the year into 183 periods (leap year) of two days. For each period has been calculated the mean values of the COVID-19 measures described in Section 3. The raw COVID-19 measures dataset can take integer values between 0 (no measures) to 4 (strict restriction). 
These values have been normalized in range [0-no measure, 1-strict measure], over a day-by-day period calculation (average).

The pollutant concentrations of the most harmful gases $\left(\mathrm{CO}, \mathrm{O}_{3}, \mathrm{NO}_{2}\right.$ and $\left.\mathrm{SO}_{2}\right)$ has been downloaded from Sentinel-5P/TROPOMI satellite from Google Earth Engine API [24]. To be more specific only the density band, which represent the density of pollutant per pixel in $\mathrm{mol} / \mathrm{m}^{2}$ unit has been downloaded. As clipped geometry has been chosen a square of $0.50 \mathrm{deg}$ with center coordinates the longitude and latitude of the city center, with a pixel size of $50 \mathrm{~m} \times 50 \mathrm{~m}$. The final pixel density has been calculated as the mean density per period. Furthermore for the linear regression to run the image needed to be transformed as a scalar value. The transformation achieved by calculated the mean and standard deviation per city for each of the four pollutants.

Finally, the two datasets combined and for each country the train/test input compromised from the 8 COVID-19 measures and one pollutant, out of four in total, (mean and standard deviation for the current week), while the train/test output compromised from the mean and standard deviation for the next week. In the end, experiments were based on a dataset of size 10 feature values $\times 182$ periods $\times 4$ cities. The same dataset has been, also, used to run the correlation tests.

\subsection{Experimental results}

Figure 1 indicates the coefficient of determination $(\mathrm{CoD})$. The first observation is that all measures have $C o D<20 \%(0.20)$, which indicates weak to moderate correlation for most cases. In particular, internal travel restrictions had a moderate correlation to NO2 levels. Yet, the same restrictions appear to have no correlation to $C O$ levels. Restrictions of Internal Movement seems to have no correlation with any of the pollutant factors' levels.

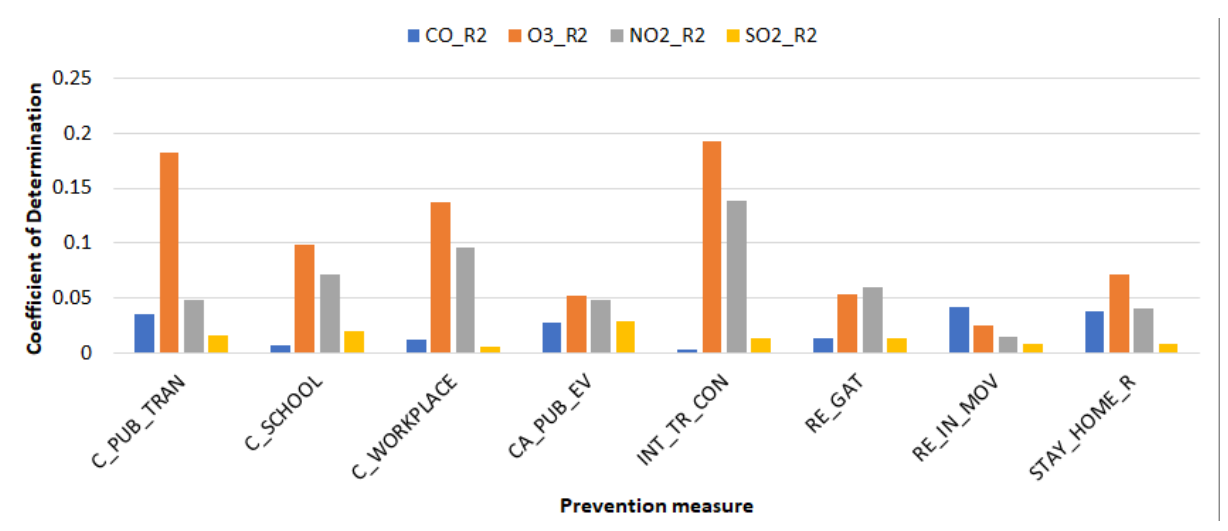

Figure 1. Coefficient of Determination $\left(R^{2}\right)$ from Pearson Correlation

Figure 2 presents the alignment distance between the investigated pollutant factor and the applied measure. A small distance suggest that the behavioural patterns are more related. As such, closing the public transportation or imposing restrictions in movements signal patterns are closer to the ones of the pollutant factors. In this case the alignment distance was at least two times lesser compared to the one of international travel controls. The same is observed for the restriction in gatherings. 


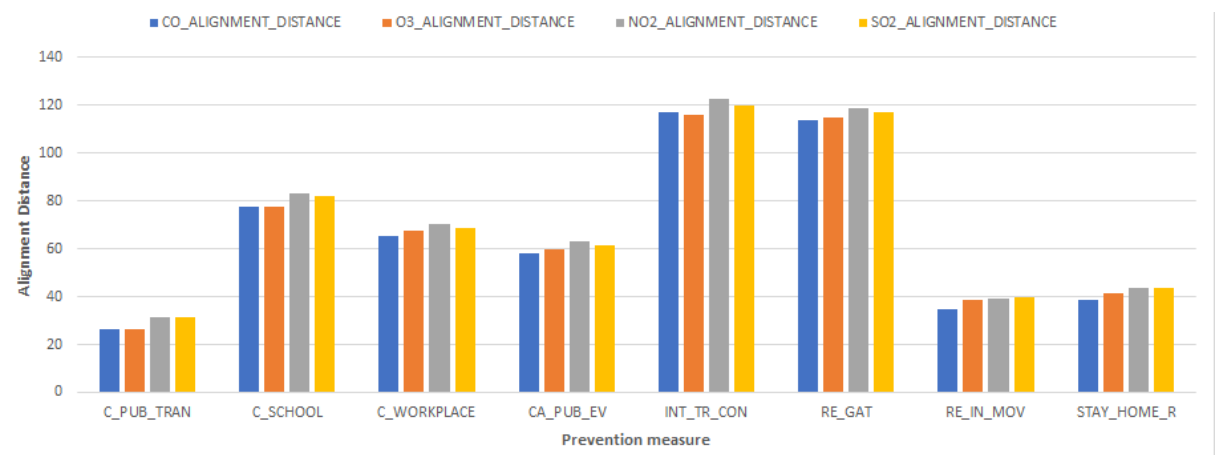

Figure 2. Alignment Distance from Dynamic Time Warping

Taking into account the previous analysis, we decided to use all measures for the training/testing the machine/deep learning techniques. From Figure 3 we can safely assume that RFR performs slightly better than the other techniques for both Carbon Monoxide $(\mathrm{CO})$ and Ozone $\left(\mathrm{O}_{3}\right)$. Especially the DNN has the worst results for predicting the Ozone. In case of Nitrogen Dioxide and Sulphur Dioxide prediction (Figure 4), the best results observed in MAdaB technique. In this point we need to specify that the errors maybe seems low, however the difference with the input values (pollutant gases concentration) is in a $10 \%$ scale (e.g. if the original value was 0.000030 the output value could be 0.000028 , in case of $\mathrm{NO}_{2}$ ).

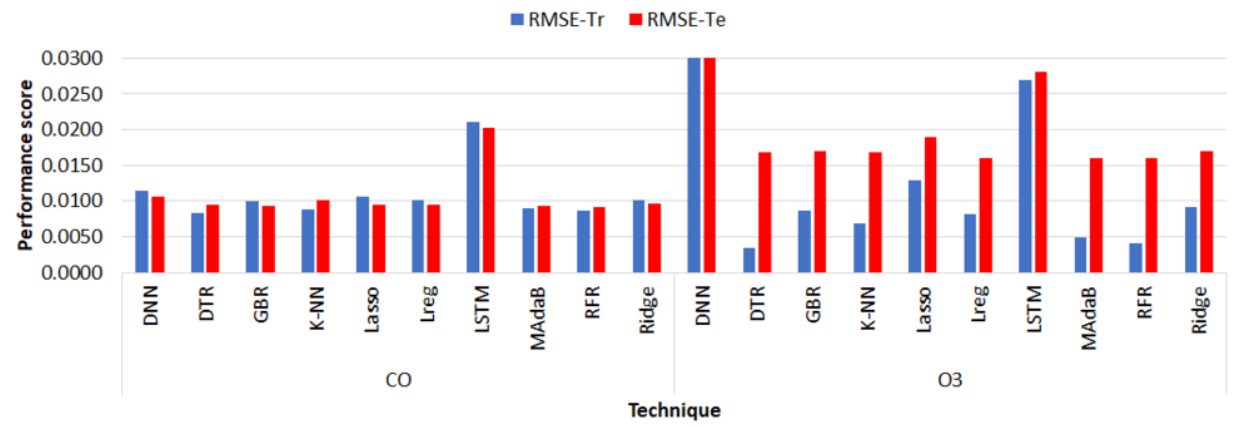

Figure 3. Root Mean Square Error (RMSE) for each machine and deep learning techniques for $\mathrm{CO}_{\text {and }} \mathrm{O}_{3}$.

\section{Conclusions}

In this work we indicated that a weak correlation between the COVID-19 and the behaviour of the four most dangerous pollutant gases exists. To prove this correlation we performed a series of tests using Pearson and Dynamic Time Warping techniques for each of the 4 European Cities, using the each day-by-day period COVID-19 measure values and each pollutant gas density. Figures 1 and 2, which depict the coefficient of determination and the alignment distance, respectively, show that there is no measure with strong correlation, thus we used all the measures for the machine and deed learning models. 


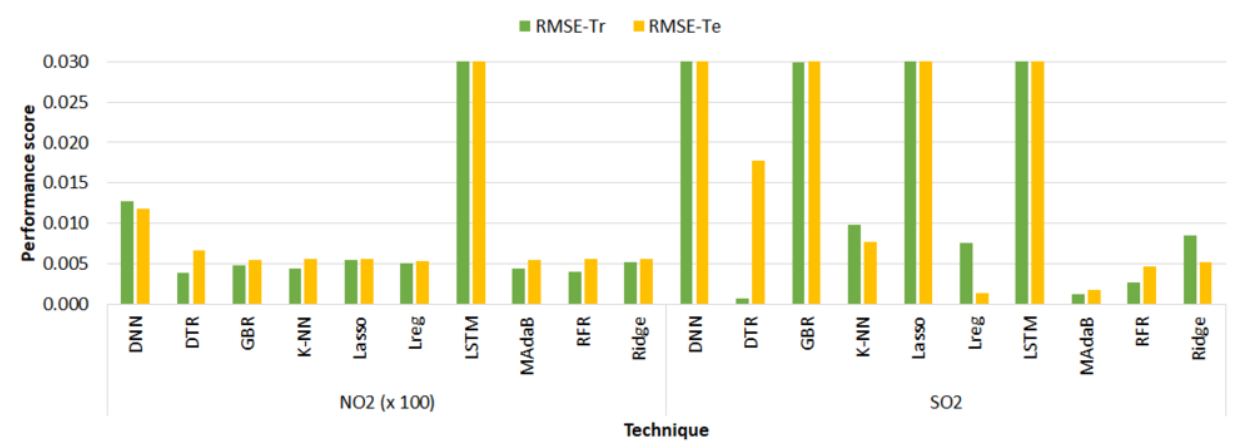

Figure 4. Root Mean Square Error (RMSE) for each machine and deep learning techniques for $\mathrm{NO}_{2}$ and $\mathrm{SO}_{2}$.

The machine learning outputs were sufficient enough for future predictions of the densities for each pollutant gas in a two days period. However, using bigger dataset the results can be further improved. Models like these, can be used, in the near future, for the estimation of the benefits from the replacement of petrol and oil vehicles, with other environmental friendly vehicles.

\section{Acknowledgement}

This paper is supported by the European Union Funded project euPOLIS "Integrated NBS-based Urban Planning Methodology for Enhancing the Health and Well-being of Citizens: the euPOLIS Approach" under the Horizon 2020 program H2020-EU.3.5.2., grant agreement No 869448.

\section{References}

[1] World Health Organization. 2020. Events as they happen, https://www. who.int/emergencies/ diseases/novel-coronavirus-2019/events-as-they-happen

[2] Ramadhan Tosepu, Joko Gunawan, Devi Savitri Effendy, La Ode Ali Imran Ahmad,Hariati Lestari, Hartati Bahar, and Pitrah Asfian, (2020) Correlation betweenweather and Covid-19 pandemic in Jakarta, Science of The Total Environment 725, Indonesia. (2020), 138436. https://doi.org/10.1016/j . scitotenv . 2020.138436

[3] Hannah Ritchie, Esteban Ortiz-Ospina, Diana Beltekian, Edouard Mathieu, JoeHasell, Bobbie Macdonald, Charlie Giattino, and Max Roser, (2020), Policy Responses to the Coronavirus Pandemic. https://ourworldindata.org/policy-responses-covid

[4] Shubham Sharma, Mengyuan Zhang, Anshika, Jingsi Gao, Hongliang Zhang, and Sri Harsha Kota, (2020) Effect of restricted emissions during COVID-19on air quality in India. Science of The Total Environment 728 (2020), 138878. https://doi.org/10.1016/j.scitotenv.2020.138878

[5] Sneha Gautam and Luc Hens, (2020) SARS-CoV-2 pandemic in India: what might we expect? Environment, Development and Sustainability 22 (2020), 3867-3869. https://doi.org/10.1007/ s10668-020-00739-5

[6] Liane Yuri, Kondo Nakada and Rodrigo Custodio Urban, (2020) COVID-19 pan-demic: Impacts on the air quality during the partial lockdown in São Paulostate, Brazil. Science of The Total Environment 730 (2020), 139087. https://doi.org/10.1016/j.scitotenv.2020.139087

[7] Aurelio Tobías, Cristina Carnerero, Cristina Reche, Jordi Massagué, Marta Via, María Cruz Minguillón, Andrés Alastuey, and Xavier Querol, (2020) Changesin air quality during the lockdown in Barcelona (Spain) one month into the SARS-CoV-2 epidemic. Science of The Total Environment 726 (2020), 138540. https://doi.org/10.1016/j.scitotenv.2020.138540 
[8] Tengku Azman, Tengku Mohd, Mohd Khair Hassan, and Wmk Aziz, (2015) Mathematical Modeling and Simulation of an Electric Vehicle. International Journal of Mechanical Sciences 8 (2015, July), 1312-1321. http://dx.doi.org/10.15282/jmes.8.2015.6.0128

[9] Enrico Ferrero, Stefano Alessandrini, and Alessia Balanzino, (2016) Impact of the electric vehicles on the air pollution from a highway. Applied Energy 169 (2016), 450-459. https : //doi .org/10.1016/ j.apenergy . 2016.01.098

[10] Guojun He, Yuhang Pan, and Takanao Tanaka, (2020) The short-term impacts of COVID-19 lockdown on urban air pollution in China. Nature Sustainability 3 (122020), 1005-101. https://doi.org/ 10.1038/s41893-020-0581-y

[11] Guojun He, Yuhang Pan, and Takanao Tanaka, (2020) COVID-19, City Lockdowns, and Air Pollution: Evidence from China medRxiv (2020), 39. https : //doi .org/10.1101/2020.03.29.20046649

[12] Chanchan Gao, Shuhui Li, Min Liu, Fengying Zhang, V. Achal, Yue Tu, ShiqingZhang, and Chaolin Cai, (2021) Impact of the COVID-19 pandemic on air pollution in Chinese megacities from the perspective of traffic volume and meteorological factors 773 (2021), 145545. https://doi.org/10.1016/j. scitotenv.2021.145545

[13] Lijuan Shen, Tianliang Zhao, Honglei Wang, Jane Liu, Yongqing Bai, ShaofeiKong, Huang Zheng, Yan Zhu, and Zhuozhi Shu, (2021) Importance of meteorology in air pollution events during the city lockdown for COVID-19 in Hubei Province Central China. Science of The Total Environment 754 (2021), 142227. https://doi.org/10.1016/j.scitotenv.2020.142227

[14] Vikas Singh, Shweta Singh, Akash Biswal, Amit P. Kesarkar, Suman Mor, andKhaiwal Ravindra, (2020) Diurnal and temporal changes in air pollution during COVID-19 strict lockdown over different regions of India. Environmental Pollution 266 (2020), 115368. https://doi.org/10.1016/j. envpol.2020.115368

[15] Khurram Shehzad, Muddassar Sarfraz, and Syed Ghulam Meran Shah, (2020) The impact of COVID-19 as a necessary evil on air pollution in India during the lockdown. Environmental Pollution 266 (2020), 115080. https://doi .org/10.1016/j.envpol.2020.115080

[16] Susanta Mahato, Swades Pal, and Krishna Gopal Ghosh, (2020) Effect of lockdown amid COVID-19 pandemic on air quality of the megacity Delhi, India. Science of The Total Environment 730 (2020), 139086. https://doi.org/10.1016/j.scitotenv.2020.139086

[17] Aviral Agarwal, Aman Kaushik, Sankalp Kumar, and Rajeev Kumar Mishra, (2020) Comparative study on air quality status in Indian and Chinese cities before and during the COVID-19 lockdown period. Air Quality, Atmosphere \& Health 13, 10 (Oct. 2020), 1167-1178. https://doi.org/10.1007/ s11869-020-00881-z

[18] Ioannis Kavouras, Maria Kaselimi, Eftychios Protopapadakis, Nikolaos DOulamis, (2021) Machine Learning Tools to Assess the Impact of COVID-19 CivilMeasures in Atmospheric Pollution (2021). https://doi.org/10.1145/3453892.3461327

[19] José M. Baldasano, (2020) COVID-19 lockdown effects on air quality by NO2 in the cities of Barcelona and Madrid (Spain). Science of The Total Environment 741 (2020), 140353. https://doi.org/10. 1016/j.scitotenv.2020.140353

[20] Bassim Mohammed Hashim, Saadi K. Al-Naseri, Ali Al-Maliki, and Nadhir Al-Ansari, (2021) Impact of COVID-19 lockdown on $\mathrm{NO}_{2}, \mathrm{O}_{3}, \mathrm{PM}_{2.5}$ and $\mathrm{PM}_{10}$ concentrations and assessing air quality changes in Baghdad, Iraq. Science of The Total Environment 754 (2021), 14197. https : //doi .org/10.1016/ j.scitotenv.2020.141978

[21] Ioannis Kavouras, Eftychios Protopapadakis, Anastasios Doulamis, and Nikolaos Doulamis, (2018) Skeleton extraction of dance sequences from 3D points using convolutional neural networks based on a new developed C3D visualization interface. In International Conference on Interactive Collaborative Learning Springer, 267-279. https : //doi .org/10.1007/978-3-030-11935-5_26

[22] Eftychios Protopapadakis, Athanasios Voulodimos, and Nikolaos Doulamis, (2018) Multidimensional Trajectory Similarity Estimation via Spatial-Temporal Keyframe Selection and Signal Correlation Analysis. In Proceedings of the 11th PErvasive Technologies Related to Assistive Environments Conference (PETRA '18). Association for Computing Machinery, New York, NY, USA, 91-97. https: //doi.org/10.1145/3197768.3201533

[23] George Kopsiaftis, Eftychios Protopapadakis, Athanasios Voulodimos, Nikolaos Doulamis, and Aristotelis Mantoglou, (2019) Gaussian process regression tuned by bayesian optimization for seawater intrusion prediction. Computational intelligence and neuroscience 2019 (2019). https : //doi . org/10. $1155 / 2019 / 2859429$ 
[24] Earth Engine Data Catalog, n.d. Sentinel-5P. https://developers.google.com/earth-engine/ datasets/catalog/sentinel-5p

[25] Zhong Shaobo,Yu Zhichen, Zhu Wei, (2019) "Study of the Effects of Air Pollutants on Human Health Based on Baidu Indices of Disease Symptoms and Air Quality Monitoring Data in Beijing, China” Int. J. Environ. Res. Public Health 16, no. 6, 1014. https ://doi .org/10.3390/i jerph16061014

[26] Xia Zhi, Song Yuexin, Ma Jin, Zhou Lujie, and Dong Zijian, (2017) Research on the Pearson correlation coefficient evaluation method of analog signal in the process of unit peak load regulation. In 2017 13th IEEE International Conference on Electronic Measurement Instruments (ICEMI), 522-527. https: //doi.org/10.1109/ICEMI.2017.8265997

[27] Müller, Meinard, (2007), Dynamic Time Warping. Springer Berlin Heidelberg, Berlin, Heidelberg, 69-84. https://doi.org/10.1007/978-3-540-74048-3_4

[28] L. Deng, G. Hinton, and B. Kingsbury, (2013) New types of deep neural network learning for speech recognition and related applications: an overview. In2013IEEE International Conference on Acoustics, Speech and Signal Processing (2013), 8599-8603. https ://doi .org/10.1109/ICASSP. 2013. 6639344

[29] Pratap Sen, Mahimarnab Hajra, and Mitadru Ghosh, (2020) Supervised Classification Algorithms in Machine Learning: A Survey and Review, 99-111. https : //doi .org/10.1007/978-981-13-7403-6_ 11

[30] Beckmann M., Ebecken N., and Pires de Lima B, (2015) A KNN Undersampling Approach for Data Balancing. Journal of Intelligent Learning Systems and Applications 7, 104-116. https : //doi .org/ 10.4236/jilsa.2015.74010

[31] Ryan J., Tibshirani, (2012) The Lasso Problem and Uniqueness. arXiv:1206.0313 [math.ST]

[32] (2020) Adaptive boosting of weak regressors for forecasting of crop production considering climatic variability: An empirical assessment. Journal of King Saud University - Computer and Information Sciences 32, 8 (2020), 949-964. https : //doi .org/10.1016/j . jksuci . 2017.12.004

[33] Paul F. Smith, Siva Ganesh, and Ping Liu, (2013) A comparison of random forest regression and multiple linear regression for prediction in neuroscience. Journal of Neuroscience Methods 220, 1 (2013), 85-91. https://doi.org/10.1016/j.jneumeth.2013.08.024

[34] Yunjie Wang, Yifan Wen, Yue Wang, Shaojun Zhang, K. Max Zhang, HaotianZheng, Jia Xing, Ye Wu, and Jiming Hao, (2020) Four-Month Changes in Air Quality during and after the COVID-19 Lockdown in Six Megacities in China. Environmental Science \& Technology Letters 7, 11 (Nov. 2020), 802-808. https://doi.org/10.1021/acs.estlett.0c00605Publisher: AmericanChemicalSociety .

[35] S. Hu, Q. Wang, J. Wang, S. S. M. Chow, and Q. Zou, (2016) Securing Fast Learning!Ridge Regression over Encrypted Big Data. In 2016 IEEE Trustcom/BigDataSE/ISPA, 19-26. https://doi.org/10. 1109/TrustCom.2016.0041

[36] Kaselimi, M., Doulamis, N., Voulodimos, A., Protopapadakis, E. and Doulamis, A., (2020) Context aware energy disaggregation using adaptive bidirectional LSTM models. IEEE Transactions on Smart Grid, 11(4), pp.3054-3067. https ://doi.org/10.1109/TSG.2020.2974347 